\title{
A new limit equilibrium method for the pseudostatic design of embedded cantilevered retaining walls
}

\author{
Riccardo Conti ${ }^{\mathrm{a}}$, Giulia M.B. Viggiani ${ }^{\mathrm{b}, *}$ \\ a SISSA, Trieste, Italy \\ ${ }^{\mathrm{b}}$ Dipartimento di Ingegneria Civile, Università di Roma Tor Vergata, Via del Politecnico, 1, 00133 Roma, Italy
}

\section{A R T I C L E I N F O}

\section{Article history:}

Received 10 May 2012

Received in revised form

6 February 2013

Accepted 18 March 2013

Available online 12 April 2013

Keywords:

Pseudostatic limit equilibrium

Earthquake

Embedded retaining walls

\begin{abstract}
A B S T R A C T
This paper describes a new pseudostatic limit equilibrium method for the design of cantilevered retaining walls under seismic actions. The method has been applied in a parametric study of the effects of the geometry of the wall, considering different excavated and embedded depths, of the strength of the soil, and of the contact between the soil and the wall. The pseudostatic predictions are in very good agreement, both in terms of horizontal contact stress and bending moment distributions, with the results of truly dynamic 2-D finite difference analyses and published experimental data. It is found that for increasing strengths of the soil-wall system both the critical acceleration and the maximum bending moment on the wall increase. In other words, a stronger soil-wall system will experience smaller displacements during the earthquake, but this is paid for by increasing internal forces in the wall.
\end{abstract}

(c) 2013 Elsevier Ltd. All rights reserved.

\section{Introduction}

The seismic design of embedded cantilevered retaining walls is conventionally carried out using a pseudostatic approach, in which dynamic actions are represented as static forces proportional to an equivalent acceleration $a_{h}=k_{h} g$, and the performance of the system is quantified in terms of a static safety factor against an assumed collapse mechanism. If $k_{h}$ is the pseudostatic coefficient related to the design earthquake, this method consists in: (i) computing the corresponding dynamic increment of the active soil pressures on the retained side of the wall, (ii) ensuring that the available reduced soil passive resistance on the excavated side is sufficient to guarantee the equilibrium of the wall (geotechnical design) and (iii) that the resulting internal forces on the structure do not exceed an assigned limit value (structural design). A more rational approach consists in admitting that limit equilibrium conditions into the soil may be exceeded during the design earthquake, and evaluating the performance of the structure in terms of permanent displacements accumulated by the wall $[4,8,7]$. Following this method, permanent displacements may be computed in a manner similar to the Newmark [17] sliding block procedure $[19,24]$.

In both simplified approaches, the critical acceleration, $a_{c}=k_{c} g$, corresponding to which the strength of the soil is fully mobilised,

\footnotetext{
*Corresponding author. Tel.: + 39 672597054; fax: + 39672597005 .

E-mail addresses: rconti@sissa.it, riccardo.conti@uniroma2.it (R. Conti), viggiani@uniroma2.it (G.M.B. Viggiani).
}

is a key ingredient: in the former method, $a_{c}$ provides an upper limit value for the maximum internal forces that the wall may ever experience during an earthquake [4] while in the latter method, $a_{c}$ is the threshold acceleration adopted in double integration procedures or simplified expressions for the estimate of permanent wall displacements [21].

The critical acceleration is defined with respect to an assumed failure mechanism, generally a rigid rotation about a point close to the toe for cantilevered walls, and depends solely on the geometry of the system and on the strength of the soil. Three different methods are proposed in the literature to compute the critical acceleration of embedded retaining walls, all of them derived from a limit equilibrium analysis. As far as anchored sheet pile walls are concerned, Towhata and Islam [22] compute the critical acceleration assuming a translation mechanism of the wall and of the retained soil wedge, while Neelakantan et al. [16] assume a rigid rotation of the wall about the anchor system. For cantilevered walls, Callisto and Soccodato [4] compute the critical acceleration with the Blum [2] method, assuming a rigid rotation of the wall about a point close to the toe.

Experimental dynamic tests carried out on reduced scale models $[16,20,5]$ have confirmed that a Newmark type calculation may be adopted, at least qualitatively, to interpret the dynamic behaviour of embedded retaining walls, where the wall can rotate when a state of limit equilibrium is attained in the adjacent soil. As far as cantilevered walls are concerned, however, numerical results also indicate that the bending moments can be substantially larger than those computed with the Blum method in critical conditions $[4,6]$. It follows that, for cantilevered walls, standard 
limit equilibrium approaches do not provide reliable nor conservative values of the yield acceleration for a pseudostatic calculation of the internal forces.

This work is focused on the pseudo-static limit equilibrium design of embedded cantilevered retaining walls, for which both the internal forces under dynamic conditions and the critical acceleration are computed customarily with the Blum method. The main drawbacks of this method are discussed, and a new limit equilibrium approach is proposed, based on results from truly dynamic 2-D finite difference analyses [6]. Also, pseudostatic predictions are compared with experimental data obtained from dynamic centrifuge tests on a cantilever wall with dry sand backfill [12].

In the following, $\phi$ is the soil friction angle, $\delta$ is the soil-wall friction angle, $\gamma$ is the unit weight of the soil, $h$ is the excavation depth, $d$ is the embedded depth, and $d_{0}$ is the location of the pivot point; the Mononobe-Okabe method $[15,18]$ is used to compute the dynamic active $\left(K_{\mathrm{AE}}\right)$ earth pressure coefficient, while passive static $\left(K_{P}\right)$ and dynamic $\left(K_{\mathrm{PE}}\right)$ earth pressure coefficients are computed using the Lancellotta $[10,11]$ closed form solutions.

\section{Dynamic behaviour of cantilevered retaining walls}

A number of studies on the dynamic behaviour of cantilevered retaining walls are reported in the literature, most of them based on the results of centrifuge tests $[25,12,13,5]$. A general observation is that bending moments in the wall increase significantly during an earthquake, reaching permanent residual values, at the end of the shaking, significantly higher than those corresponding to static conditions. Considerable difficulties still exist in the seismic design of cantilevered retaining walls because of the complex nature of the dynamic soil-structure interaction, which can be properly addressed only by numerical modelling.

Conti et al. [6] reported results from 39 plane-strain finite difference analyses of a16 m wide and $4 \mathrm{~m}$ deep rectangular excavation, retained by a pair of cantilevered retaining walls, embedded in an ideal layer of dry sand with a thickness of $16 \mathrm{~m}$. Fig. 1 shows the grid adopted in the numerical analyses, with an extension of $80 \mathrm{~m}$, consisting of a total of 4838 elements, with a minimum size of $0.33 \mathrm{~m}$ near the walls.

The retaining walls were modelled as elastic beams, with a stiffness $E I=2.7 \times 10^{5} \mathrm{kNm}^{2} / \mathrm{m}$, connected to the grid nodes with elastic-perfectly plastic interfaces with a friction angle $\delta=20^{\circ}$.

The soil was modelled as an elastic-perfectly plastic material with Mohr-Coulomb failure criterion, with a constant friction angle $\varphi=35^{\circ}$, cohesion $c^{\prime}=0$, and density $\rho=2.04 \mathrm{Mg} / \mathrm{m}^{3}$. A standard non-associated flow rule was used, with angle of dilatancy $\psi=0$. Non-linear and hysteretic behaviour was introduced for stress paths within the yield surface through a hysteretic model available in the library of FLAC 5.0 [9], consisting in an extension to general strain conditions of the one-dimensional non-linear models that make use of the Masing [14] rules to describe the unloading-reloading behaviour of soil during cyclic loading. Fig. 2 shows a comparison between the modulus decay curve and the equivalent damping ratio of the adopted model and that suggested by Vucetic and Dobry [23].

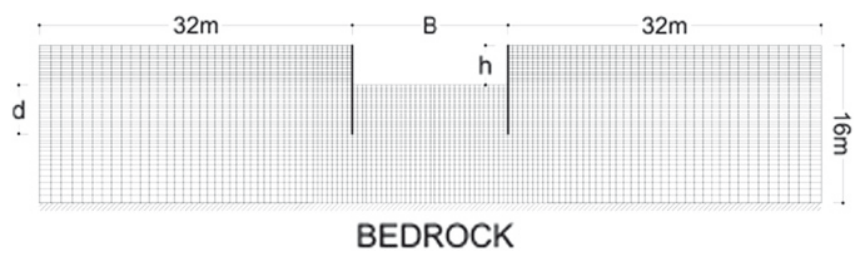

Fig. 1. Finite difference grid.

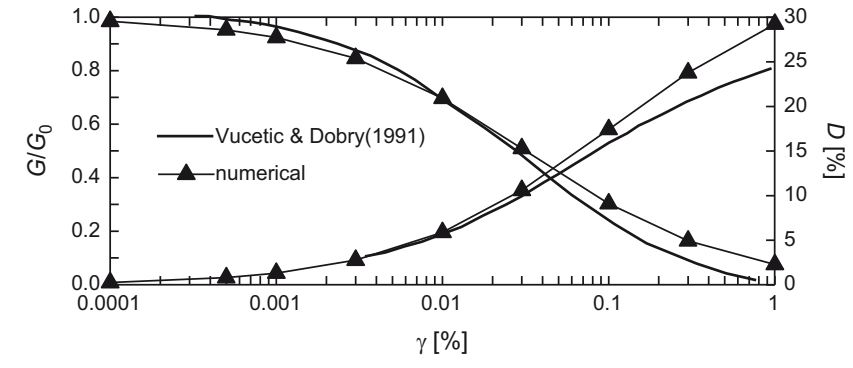

Fig. 2. Modulus decay and damping ratio curves for the constitutive soil model.

$t[\mathrm{~s}]$
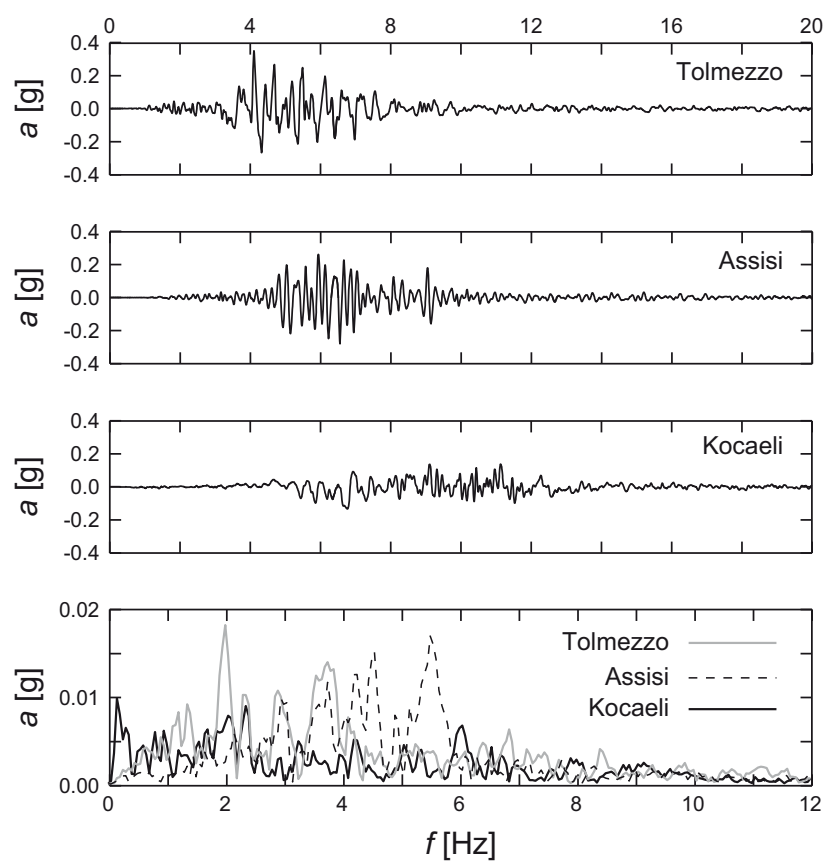

Fig. 3. Acceleration time histories and Fourier amplitude spectra of the input earthquakes.

Three different acceleration time histories (Fig. 3), all registered on rock outcrop during real earthquakes, were used in the analyses; the input signals were scaled at maximum accelerations ranging from $0.05 \mathrm{~g}$ to $0.05 \mathrm{~g}$. After the static stage, the horizontal acceleration time histories were applied to the bottom nodes of the grid, together with a zero velocity condition in the vertical direction, while standard periodic constraints [26] were applied to the lateral boundaries.

Based on the results of these analyses, the main observations can be summarised as follows:

(i) the passive resistance of the soil in front of the wall is mobilised progressively during the earthquake, starting from dredge level downwards: the stronger the applied acceleration, the greater the depth down to which the passive resistance is fully mobilised;

(ii) in the instant of time when the acceleration behind the wall reaches its maximum value, the distribution of accelerations into the soil is not uniform, due to both amplification phenomena and phase shift between the top and the bottom of the wall; moreover, the accelerations in the soil below dredge level are only a small fraction of the maximum value computed on the retained side, and always lower than about $0.1 \mathrm{~g}$ 
(iii) permanent displacements of the wall correspond to an approximately rigid rotation around a pivot point located at a depth of between $0.8 \times d$ and $0.9 \times d$.

The mechanism by which the soil passive resistance is mobilised below dredge level is the main factor governing the dynamic behaviour of cantilevered embedded walls, where the structure can rotate when a state of limit equilibrium is attained in the adjacent soil. On the other hand, dynamic active earth pressure distribution behind the walls can be predicted reasonably with the standard Mononobe-Okabe theory [18,15], provided a suitable value for the pseudostatic coefficient is adopted, taking into account amplification phenomena into the soil. Thus, consistently with other experimental and numerical studies on cantilevered walls [1], a triangular distribution for the dynamic active contact stresses can always be assumed in pseudostatic calculations, just as in the static case.

\section{Pseudostatic approaches}

\subsection{Blum method}

An extension of the Blum [2] method to dynamic conditions is generally adopted to compute both the critical acceleration and the internal forces on the wall for $k_{h} \leq k_{c}$ (Callisto and Soccodato [4]). According to this method, the dynamic active pressure, $K_{\mathrm{AE}}$, is fully mobilised in the retained soil, down to a depth $h+d_{0}$ from the surface, where $d_{0}=0.8 \times d$ (Fig. 4); a constant fraction of the dynamic passive pressure, $K_{\mathrm{PE}} / F$, is mobilised into the soil below dredge level, while $R$ accounts globally for the distribution of earth pressures around and below the pivot point. For a given $k_{h}$, the safety factor $F$ may be computed from the moment equilibrium around the pivot point; the maximum value of the pseudostatic coefficient is $k_{c}$, which corresponds to $F=1$. The value of $0.8 \times d$ adopted for $d_{0}$ is necessary to guarantee the force equilibrium for the assumed earth pressure distribution, not explicitly taken into account in the method.

As shown by Callisto and Soccodato [4] and Conti et al. [6], the Blum method does not provide a conservative estimate of the critical acceleration, in the sense that the pseudo-static values of $M_{c}\left(=M_{\max }\left(k_{c}\right)\right)$, i.e. the maximum bending moment computed with the pseudostatic method, are about 50\% lower than the

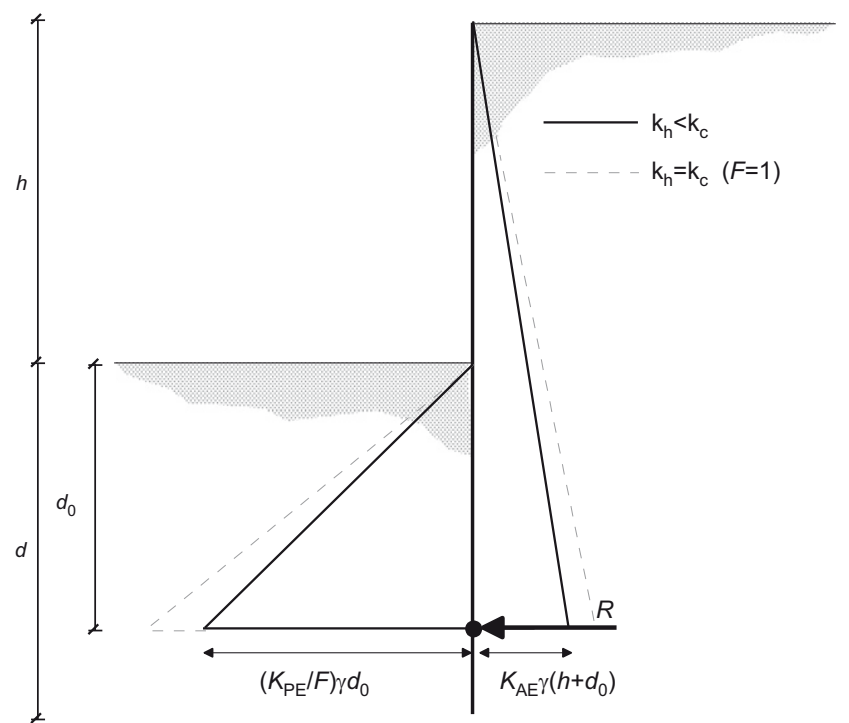

Fig. 4. Distribution of seismic earth pressures in the method by Blum [2]. values computed by numerical analyses. This arises from two main factors: in the Blum method (i) the same pseudo-static acceleration is assumed for the soil in front and behind the wall and (ii) the pivot point is assumed to be at a depth of $0.8 \times d$ from dredge level. Both assumptions lead to an underestimation of the soil passive resistance that can be effectively mobilised in front of the wall during an earthquake.

\subsection{Proposed method}

For a given $k_{h}<k_{c}$, the horizontal earth pressure distribution assumed in the proposed method is shown in Fig. 5 (continuous line). On the retained side, the soil is in active limit state down to $d_{0}$, and in passive limit state below the rotation point. On the excavated side, the passive resistance of the soil is fully mobilised down to a depth $\bar{d}$; the soil is in active limit state below the pivot point, and the horizontal contact stress decrease linearly with depth between $\bar{d}$ and $d_{0}$. To account for the numerical evidence that the accelerations below dredge level are always much smaller than those computed on the retained part of the soil, it is assumed that the passive earth pressure coefficient takes its static value, $K_{\mathrm{P}}$. This assumption is clearly conservative from the point of view of the structural design of the wall, but the difference between $K_{P}$ and $K_{\mathrm{PE}}\left(k_{h}=0.1\right)$ is lower than $5 \%$.

The force equilibrium of the wall can be established by considering the force acting on the right hand side $\left(F_{\mathrm{RHS}}\right)$ and on the left hand side $\left(F_{\mathrm{LHS}}\right)$ of the wall:

$$
\begin{aligned}
& F_{\mathrm{RHS}}=\frac{1}{2} \gamma K_{A E}\left(h+d_{0}\right)^{2}+\frac{1}{2} \gamma K_{P}\left(2 h+d+d_{0}\right)\left(d-d_{0}\right) \\
& F_{\mathrm{LHS}}=\frac{1}{2} \gamma K_{P} \bar{d}^{2}+\frac{1}{2} \gamma\left(K_{P} \bar{d}+K_{A E} d_{0}\right)\left(d_{0}-\bar{d}\right)+\frac{1}{2} \gamma K_{A E}\left(d+d_{0}\right)\left(d-d_{0}\right)
\end{aligned}
$$

Similarly, the moment equilibrium can be established by taking the moment about the toe of the wall, generated by the forces acting on the right hand side $\left(M_{\mathrm{RHS}}\right)$ and on the left hand side $\left(M_{\mathrm{LHS}}\right)$ :

$M_{\mathrm{RHS}}=\frac{1}{2} \gamma K_{A E}\left(h+d_{0}\right)^{2}\left[\frac{1}{3}\left(h+d_{0}\right)+\left(d-d_{0}\right)\right]+\frac{1}{6} \gamma K_{P}\left(3 h+d+2 d_{0}\right)\left(d-d_{0}\right)^{2}$

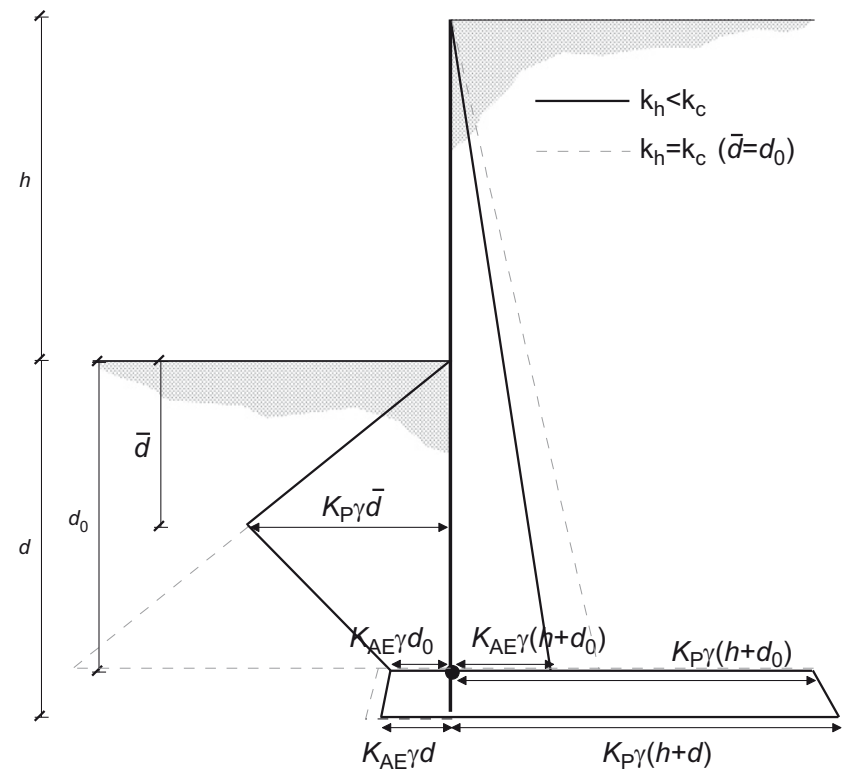

Fig. 5. Distribution of seismic earth pressures in the proposed method. 


$$
\begin{aligned}
M_{\mathrm{LHS}}= & \frac{1}{2} \gamma K_{P} \bar{d}^{2}\left(d-\frac{2}{3} \bar{d}\right) \\
& +\frac{1}{2} \gamma\left(K_{P} \bar{d}+K_{A E} d_{0}\right)\left(d_{0}-\bar{d}\right)\left[\frac{\left(d_{0}-\bar{d}\right)}{3} \frac{\left(K_{A E} d_{0}+2 K_{P} \bar{d}\right)}{\left(K_{A E} d_{0}+K_{P} \bar{d}\right)}+\left(d-d_{0}\right)\right] \\
& +\frac{1}{6} \gamma K_{A E}\left(d-d_{0}\right)^{2}\left(d+2 d_{0}\right)
\end{aligned}
$$

By equating $F_{\mathrm{RHS}}=F_{\mathrm{LHS}}$ and $M_{\mathrm{RHS}}=M_{\mathrm{LHS}}$ one obtains a system of two equations in the two unknowns $\bar{d}$ and $d_{0}$.

Fig. 6(a) shows the ratios $\bar{d} / d$ and $d_{0} / d$ as a function of $k_{h} / k_{\mathrm{c}}$, for three different values of the ratio $h / d\left(\phi=35^{\circ}, \delta=20^{\circ}, \gamma=20 \mathrm{kN} / \mathrm{m}^{3}\right.$, $h=4 \mathrm{~m}$ ), while in Fig. 6(b) the same quantities are plotted in a dimensional form. Both $\bar{d}$ and $d_{0}$ evolve with increasing $k_{h}$ : in
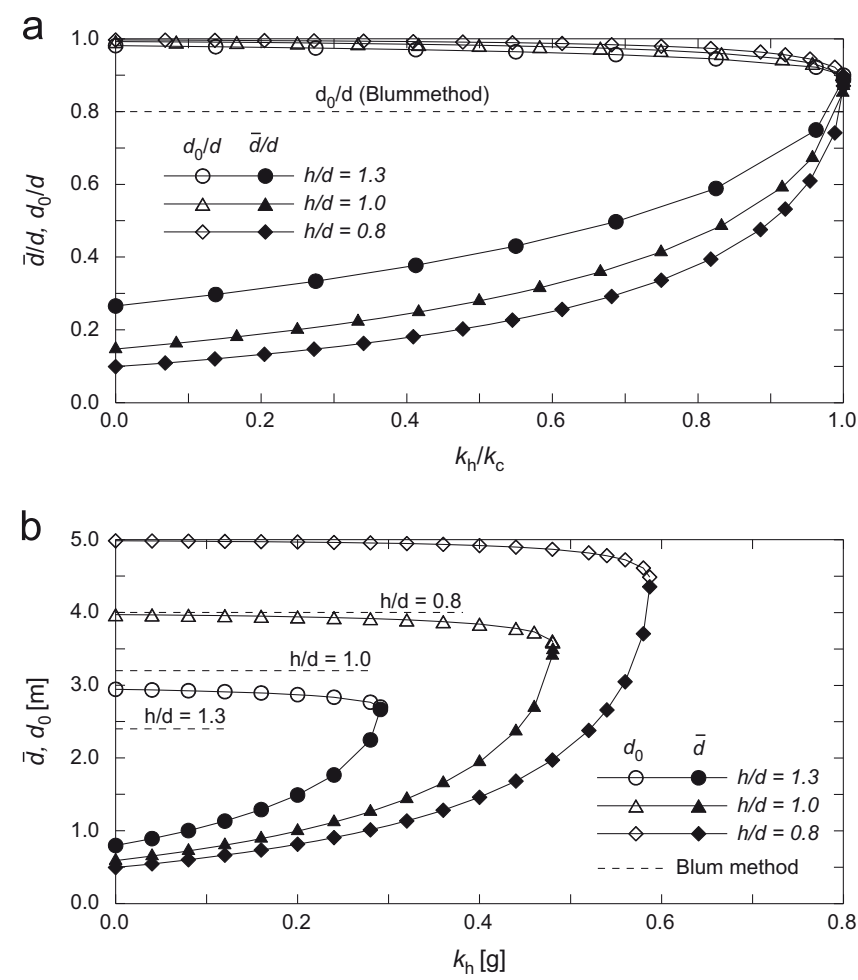

Fig. 6. Dependence of $\bar{d}$ and $d_{0}$ on the pseudostatic coefficient $k_{h}\left(\phi=35^{\circ}, \delta=20^{\circ}\right.$, $\left.\gamma=20 \mathrm{kN} / \mathrm{m}^{3}, h=4 \mathrm{~m}\right)$. static conditions $\left(k_{h} / k_{c}=0\right)$, the ratio $\bar{d} / d$ depends strongly on $h / d$ (and on the mechanical properties of both the soil and the soilwall interface), while in critical conditions $\left(k_{h} / k_{c}=1\right)$ the passive resistance of the soil below the excavation is mobilised completely down to the pivot point, i.e. $\bar{d}=d_{0}$, with $d_{0}$ about $0.9 \times d$ independently on the ratio $h / d$ (and on the mechanical properties of both soil and soil-wall interface). No further increase in the pseudostatic coefficient can take place once critical condition in the system is attained, as no more passive resistance is available in the soil in front of the wall. In the Blum method (dotted lines) the depth of the pivot point is a constant fraction of the embedment depth and does not evolve with the pseudostatic coefficient applied. As shown in Fig. 6(b), this assumption leads to values of the critical acceleration which are substantially lower than those predicted by the proposed method.

Fig. 7 shows (a) the horizontal stress and (b) the bending moment distributions computed with the proposed limit equilibrium method for different values of $k_{h}\left(\phi=35^{\circ}, \delta=20^{\circ}, \gamma=20 \mathrm{kN} /\right.$ $\mathrm{m}^{3}, h=4 \mathrm{~m}, d=4 \mathrm{~m}$ ). When $k_{h}$ increases, the dynamic active earth pressures increase in the retained soil and equilibrium of moments requires a larger fraction of the passive earth pressure to be mobilised in front of the wall. Only in critical conditions $\left(k_{h}=k_{c}\right)$ the passive earth pressure in front of the wall is fully mobilised down to the pivot point $\left(\bar{d}=d_{0}\right)$. Redistribution of earth pressures due to inertia forces into the soil results in increasing bending moments into the wall.

For comparison, Fig. 8 shows (a) the horizontal stresses and (b) the bending moment distributions computed with the Blum method for the same case reported in Fig. 7. This time the critical acceleration of the wall is $a_{c}=0.28 \mathrm{~g}$ and, accordingly, the maximum internal forces in the wall are much lower than those predicted by the proposed method. As already discussed, an increasing, but constant, fraction of the soil dynamic passive resistance is mobilised below dredge level when $k_{h}$ increases, reaching the condition $F=1$ when the critical condition is attained.

Fig. 9 shows the evolution of the normalised maximum bending moment, $M_{\max } / \gamma h^{3}$, as a function of $k_{h}$, for different values of $\phi$ and $h / d$, and for $h=4-6 \mathrm{~m}\left(\delta=1 / 3-2 / 3 \phi, \gamma=20 \mathrm{kN} / \mathrm{m}^{3}\right) . M_{\max } / \gamma h^{3}$ increases with $k_{h}$, until it reaches a limit upper value for $k_{h}=k_{c}$. For a given $k_{h}$ and $\phi, M_{\max } / \gamma h^{3}$ depends solely on the ratio $h / d$, and it increases with $d$, i.e. the longer the wall, the larger the bending moment it has to sustain. Finally, unlike what happens in static conditions, bending moments increase with increasing soil friction a

$\sigma_{\mathrm{h}}[\mathrm{kPa}]$

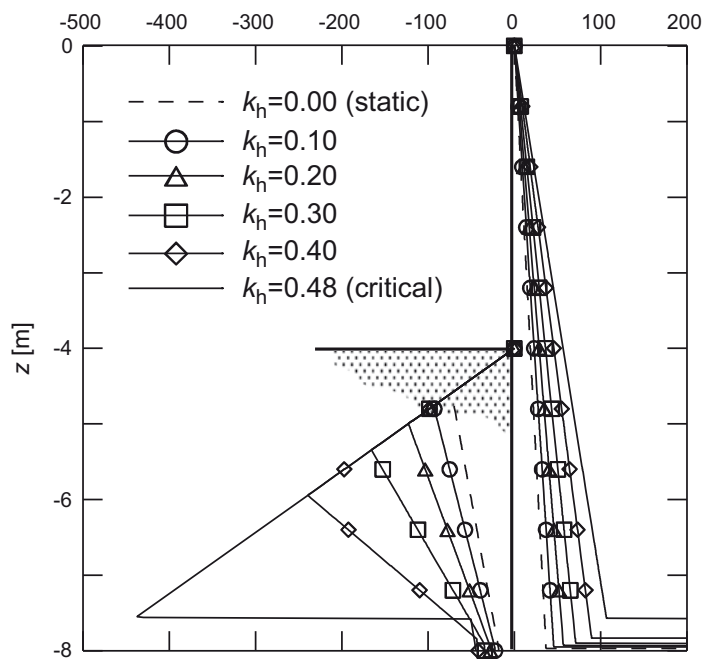

b $\quad M[K n m / m]$

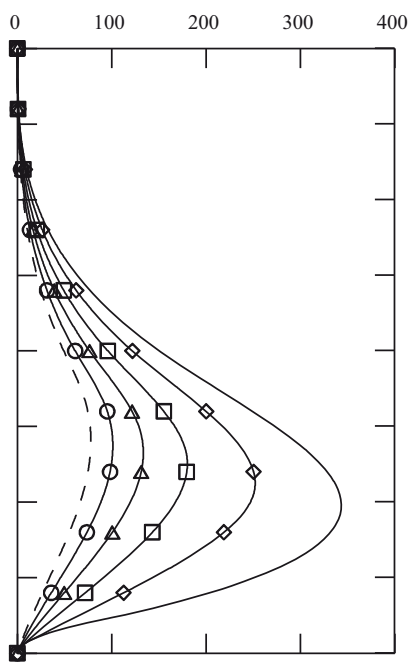

Fig. 7. Proposed method. Distribution of (a) horizontal stresses and (b) bending moment for different values of $k_{h}\left(\phi=35^{\circ}, \delta=20^{\circ}, \gamma=20 \mathrm{kN} / \mathrm{m}^{3}, h=4 \mathrm{~m}\right)$. 
a
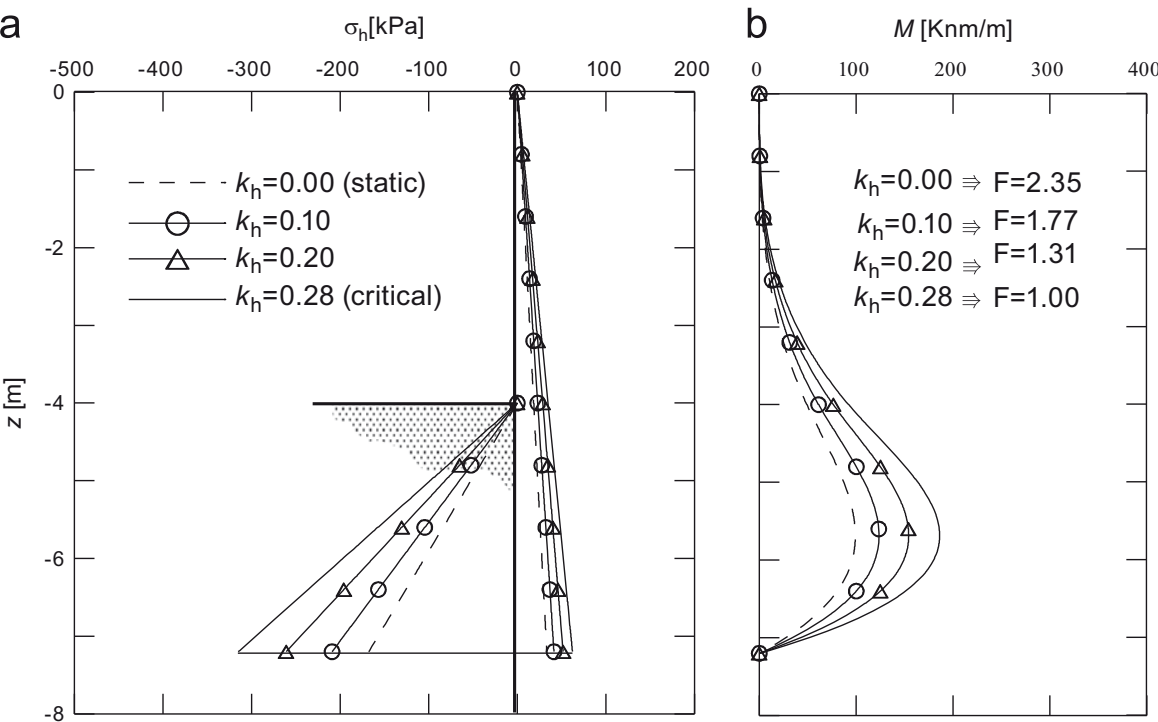

Fig. 8. Blum method. Distribution of (a) horizontal stresses and (b) bending moment for different values of $k_{h}\left(\phi=35^{\circ}, \delta=20^{\circ}, \gamma=20 \mathrm{kN} / \mathrm{m}^{3}, h=4 \mathrm{~m}\right)$.
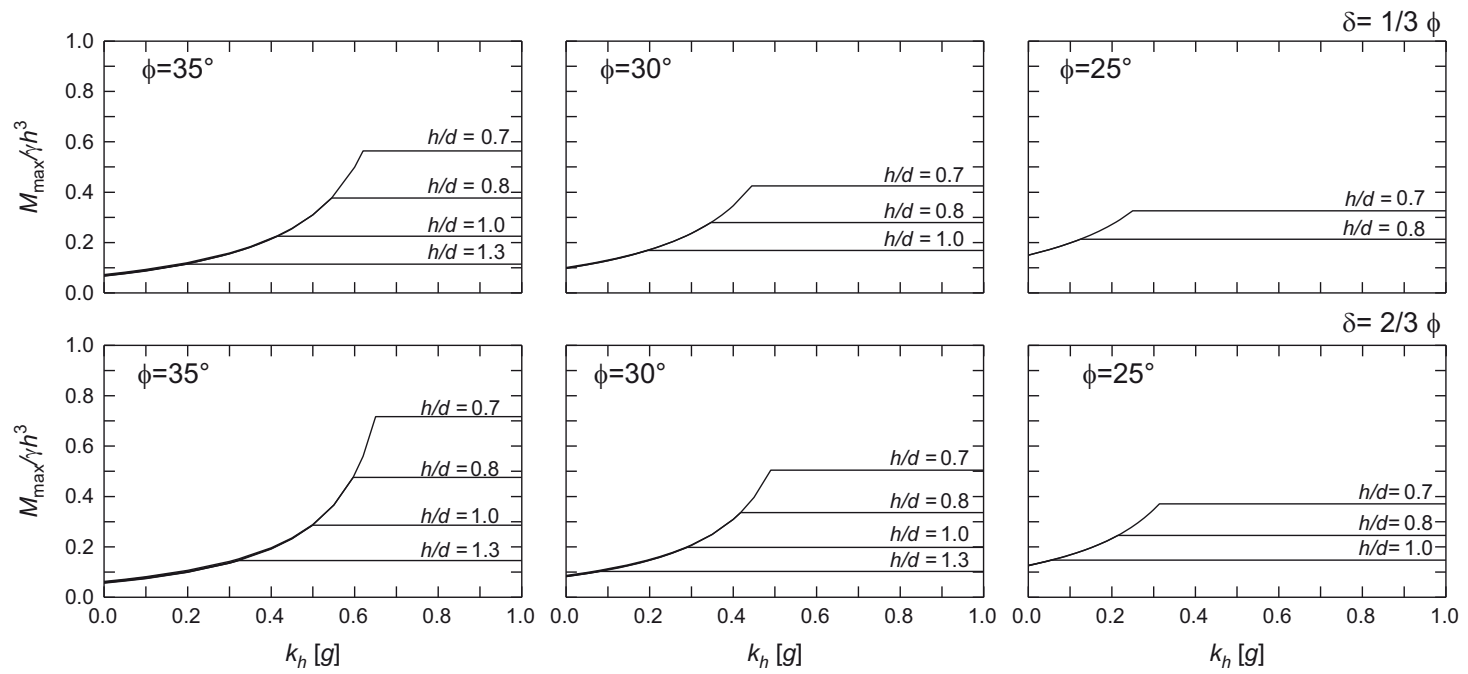

Fig. 9. Normalised maximum bending moment, $M_{\max } / \gamma h^{3}$ as a function of $k_{h}$, for different values of $\phi$ and $h / d\left(\delta=1 / 3 \phi\right.$ and $\left.\delta=2 / 3 \phi, \gamma=20 \mathrm{kN} / \mathrm{m}^{3}\right)$.

angle. These observations are more evident by inspection of Fig. 10, where the critical acceleration, $a_{\mathrm{c}}$, and the corresponding normalised maximum bending moment, $M_{\mathrm{c}} / \gamma h^{3}$, are plotted as a function of $h / d$, for different values of $\phi(\delta=1 / 3-2 / 3 \phi, \gamma=20 \mathrm{kN} /$ $\mathrm{m}^{3}$ ). The stronger the soil-wall system, that is the larger the soil friction angle and the embedded depth, the larger both $a_{\mathrm{c}}$ and $M_{\mathrm{c}}$. In other words, a stronger soil-wall system will experience smaller displacements during the earthquake, but should be designed to sustain larger internal forces.

3.3. Comparison between pseudo-static calculations, numerical data and experimental results

Fig. 11 shows a comparison between numerical results and limit equilibrium predictions of the earth pressure and the bending moment distributions on a cantilevered wall subjected to a real earthquake scaled at four different values of the maximum acceleration (analyses no. 14 to 17 in Conti et al. [6], see Table 1). The critical acceleration of the wall is equal to $a_{c}=0.28 \mathrm{~g}$, according to the Blum method, and $a_{c}=0.48 \mathrm{~g}$ according to the proposed method. Numerical data refer to the time instant when the accelerations behind the wall reach their maximum value, $a_{\max }$.
In the pseudostatic calculations, $k_{h}$ is assumed to be equal to the average value of the accelerations behind the wall, along the first $2 \mathrm{~m}$ below the soil surface, if $k_{h}<k_{c}$, while $k_{h}=k_{c}$ otherwise. The earth pressure distributions assumed in the Blum method are completely different from the numerical ones, and the method provides reliable values for the maximum bending moment in the wall only for moderate earthquakes (Fig. 11(b-d)). On the contrary, both horizontal stresses and bending moments computed with the proposed method are in good agreement with the numerical data, even if some discrepancies can be still observed (see e.g. Fig. 11 (d)), mainly due to small non-uniformities in the distribution of the accelerations into the retained soil, not taken into account in the limit equilibrium calculation.

The prediction capabilities of the proposed method are assessed also with reference to the experimental data presented by Madabhushi and Zeng [12,13], obtained from a centrifuge dynamic test carried out on a cantilevered wall model embedded in a uniform layer of dry sand. All the data presented in the following are at prototype scale.

The excavation and the embedded depths of the wall were equal to $h=7.2 \mathrm{~m}$ and $d=7.2 \mathrm{~m}$ respectively. A standard fine silica sand was used (Leighton Buzzard 52/100), reconstituted at a relative density 

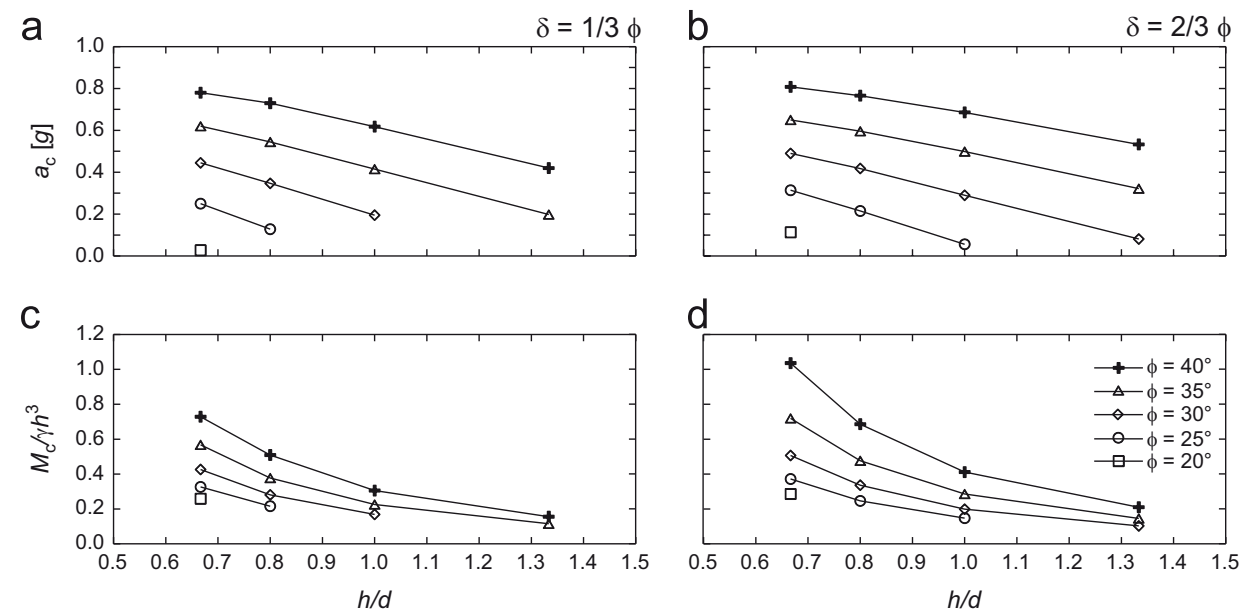

Fig. 10. Dependence of (a) and (b) critical acceleration, $a_{\mathrm{c}}$, and (c) and (d) normalised maximum bending moment, $M_{\mathrm{c}} / \gamma h^{3}$, on $h / d\left(\delta=1 / 3 \phi\right.$ and $\left.\delta=2 / 3 \phi, \gamma=20 \mathrm{kN} / \mathrm{m}^{3}\right)$.
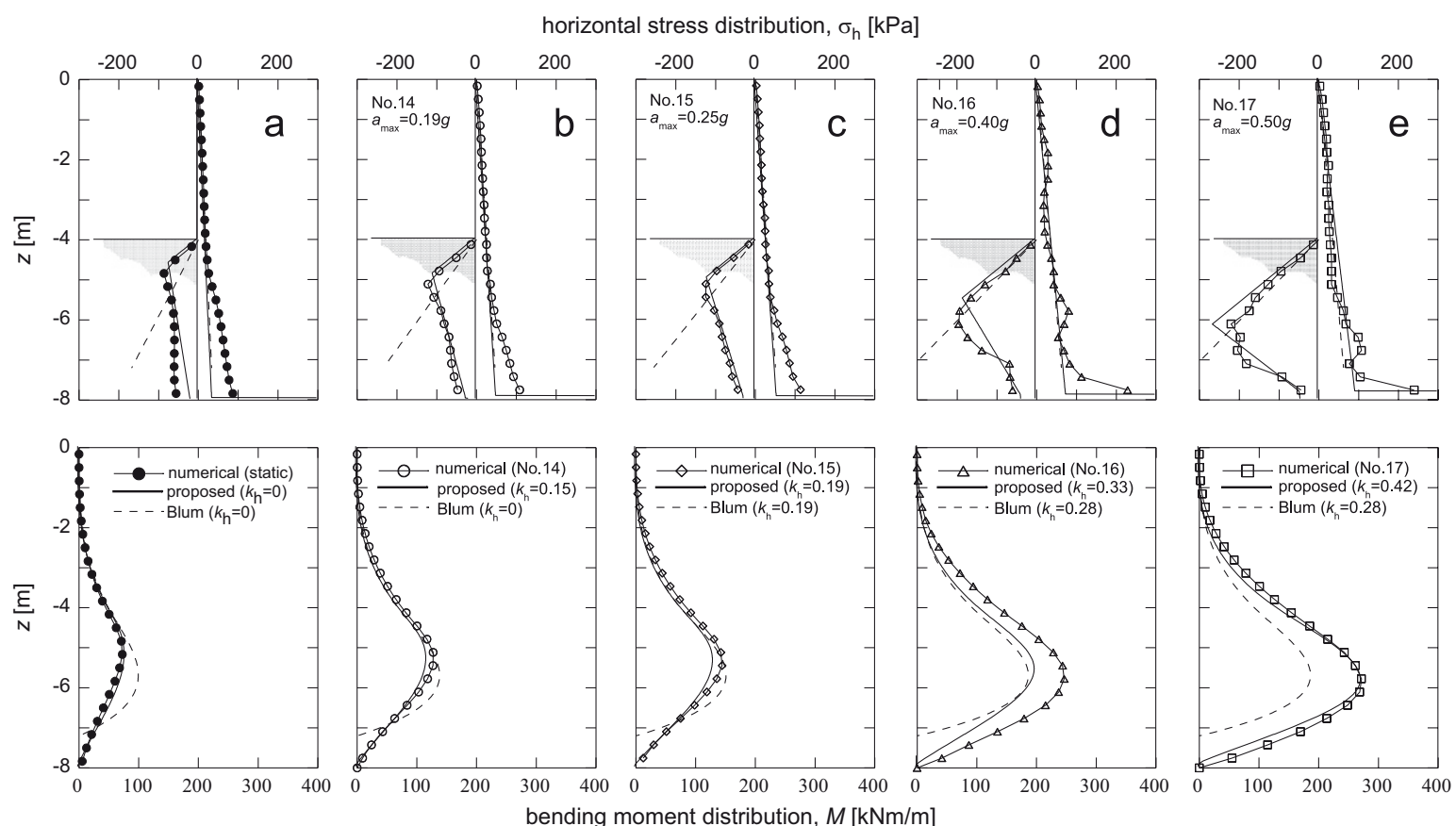

Fig. 11. Comparison between numerical results and limit equilibrium predictions for a real earthquake scaled at four different values of the maximum acceleration $\left(\phi=35^{\circ}\right.$, $\left.\delta=20^{\circ}, \gamma=20 \mathrm{kN} / \mathrm{m}^{3}, h=4 \mathrm{~m}, d=4 \mathrm{~m}\right)$.

Table 1

Summary of the numerical analyses.

\begin{tabular}{llll}
\hline No. & Input & $a_{\text {inp }}[\mathrm{g}]$ & $d[\mathrm{~m}]$ \\
\hline 14 & Tolmezzo & 0.05 & 4 \\
15 & Tolmezzo & 0.10 & 4 \\
16 & Tolmezzo & 0.20 & 4 \\
17 & Tolmezzo & 0.35 & 4 \\
\hline
\end{tabular}

$D_{R}=92 \%$ (unit weight $\gamma_{d}=16.4 \mathrm{kN} / \mathrm{m}^{3}$ ), with critical friction angle $\phi_{\mathrm{cv}}=33^{\circ}$. The model was subjected to three trains of approximately sinusoidal waves (EQ), with a nominal frequency of about $1.25 \mathrm{~Hz}$, increasing amplitudes and a constant duration of $10 \mathrm{~s}$. The maximum applied accelerations were equal to $0.12 \mathrm{~g}$ (EQ1), $0.22 \mathrm{~g}$ (EQ2) and $0.23 \mathrm{~g}$ (EQ3) respectively. For the first and the last earthquake, which will be discussed in the present paper, no significant amplifications were observed within the backfill. Fig. 12 shows a comparison between the bending moments on the wall measured in the test and the corresponding distributions predicted by the two pseudostatic approaches; for completeness, the numerical results obtained by Madabhushi and Zeng [12] are also included in the figure. Table 2 summarises the data adopted in the analyses: the value $\delta=12^{\circ}$ for the soil-wall friction angle was provided by Madabhushi and Zeng [13], while the mobilised soil friction angle was estimated taking into account both the dilatancy effects due to the high relative density of the sand [3] and the progressive shear strain mobilisation during the dynamic events.

The bending moment distribution predicted by the proposed method is in good agreement with the experimental data, both in magnitude and trend. Only for the last earthquake, the method provides a good estimation of the maximum bending moment, but at a deeper depth below dredge level. However, a similar trend can be observed for the numerical results, where the computed bending moments are lower than the centrifuge measurements, especially in the upper part of the wall. It is believed that these discrepancies can be partly due to experimental factors, such as the action exerted on the model by the electrical connexions from the strain gauges. 


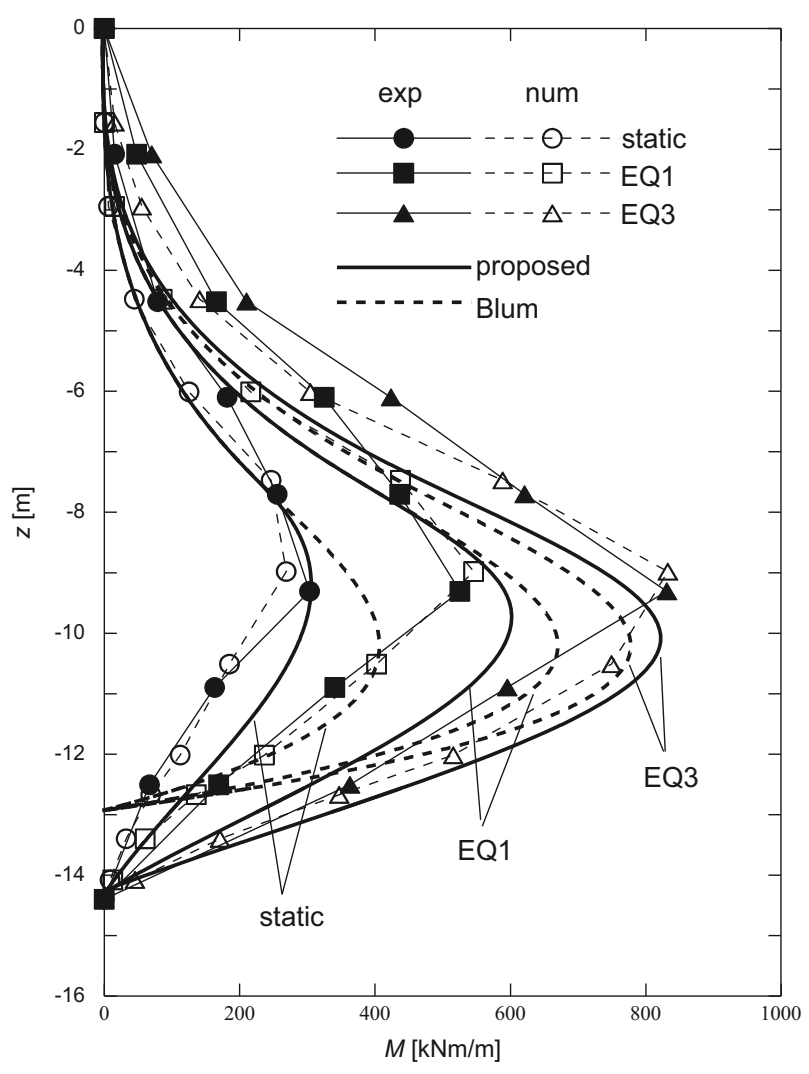

Fig. 12. Bending moment distribution on the wall: comparison between experimental data from centrifuge dynamic tests, numerical results and limit equilibrium predictions (experimental and numerical data from [12]).

Table 2

Parameters adopted for the pseudostatic analysis of the centrifuge dynamic test.

\begin{tabular}{clll}
\hline Event & $\phi\left[^{\circ}\right]$ & $k_{h}$ (proposed m.) & $k_{h}$ (Blum m.) \\
\hline static & 40 & 0.000 & 0.000 \\
EQ1 & 34 & 0.120 & 0.120 \\
EQ3 & 34 & 0.230 & 0.192 \\
\hline
\end{tabular}

On the other hand, in the Blum method the maximum bending moment is always located at the same depth, about $10 \mathrm{~m}$ from the soil surface, and the predicted values do not match with the experimental data. Moreover, the method provides a critical acceleration of $a_{\mathrm{c}}=0.192 \mathrm{~g}$, that is it predicts no further increase of the internal forces in the wall for pseudostatic coefficients higher than $k_{c}=0.192$.

A final comparison between numerical results and limit equilibrium calculations is shown in Fig. 13, where the normalised maximum bending moments on the walls (analyses no. 1 to 36 in Conti et al. [6]) are plotted against the maximum accelerations computed behind the walls during the earthquakes. As already observed, the Blum method provides a reliable estimate of the maximum internal forces on the wall only for moderate earthquakes, and underestimates substantially both the critical acceleration and the corresponding maximum bending moment. On the contrary, the proposed method is in good agreement with the numerical data and provides always conservative values of the maximum (critical) bending moment.

\section{Conclusions}

This work has illustrated a pseudostatic limit equilibrium method for the design of cantilevered retaining walls under

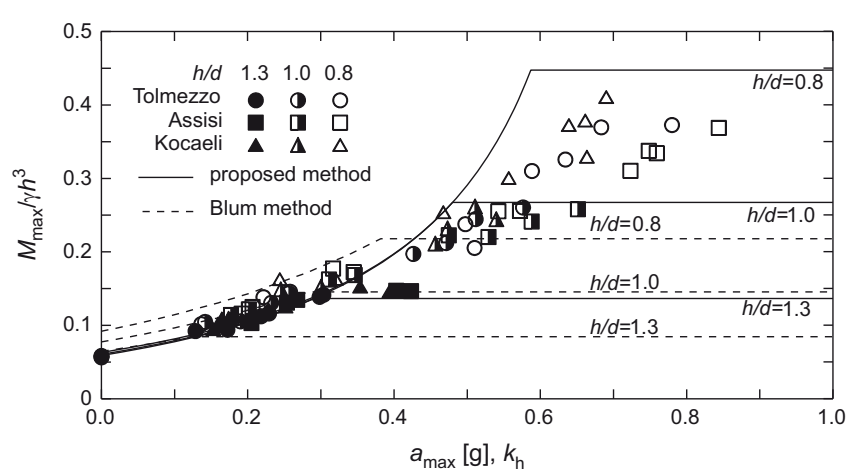

Fig. 13. Maximum normalised bending moments on embedded cantilevered walls subjected to real earthquakes, computed from dynamic numerical simulations and limit equilibrium $\left(\phi=35^{\circ}, \delta=20, \gamma=20 \mathrm{kN} / \mathrm{m}^{3}, h=4 \mathrm{~m}\right)$.

seismic actions. The method has been applied in a parametric study of the effects of the geometry of the wall, considering different excavated depths, $h$, and different ratios of the excavated and embedded depth, $h / d$, and of the strength of the soil, $\phi$, and of the contact of the soil and the wall, $\delta$. The results show that for increasing strengths of the soil-wall system, that is for increasing values of the soil and soil-wall friction angles and for increasing embedded depths, both the critical acceleration, $a_{\mathrm{c}}$, and the maximum bending moment on the wall, $M_{c}$, increase. In other words, a stronger soil-wall system will experience smaller displacements during the earthquake, but this is paid for by increasing internal forces in the wall.

The proposed limit equilibrium method has been validated against both the results of an extensive numerical study of the behaviour of cantilevered walls under seismic actions [6] and the experimental data of a centrifuge dynamic test on cantilevered wall embedded in dry sand [13]. In both cases the pseudostatic predictions have shown good agreement with the numerical and experimental findings, both in terms of magnitude and trend of the internal forces in the wall.

\section{Acknowledgements}

The work presented in this paper is part of the ReLUIS research project, funded by the Italian Department of Civil Protection.

\section{References}

[1] Atik LA, Sitar N. Seismic earth pressures on cantilevered retaining structures. Journal of Geotechnical and Geoenvironmental Engineering 2010;136 (10):1324-33.

[2] Blum H. Einspannungsverh̆altnisse bei Bohlkwerken. Berlin: Wil. Ernst und Sohn; 1931.

[3] Bolton M. The strength and dilatancy of sands. Géotechnique 1986;36 (1):65-78.

[4] Callisto L, Soccodato FM. Seismic design of flexible cantilevered retaining walls. Journal of Geotechnical and Geoenvironmental Engineering 2010;136 (2):344-54.

[5] Conti R, Madabhushi SPG, Viggiani GMB. On the behaviour of flexible retaining walls under seismic actions. Géotechnique 2012;62(12):1081-94.

[6] Conti R, Viggiani GMB, Burali d'Arezzo F. Some remarks on the seismic behaviour of embedded cantilevered retaining walls, submitted to Géotechnique; 2013.

[7] Iai S. Seismic performance-based design of port structures and simulation techniques. In: Proceedings of NSF international workshop on earthquake simulation in geotechnical engineering. Case Western Reserve University, Cleveland; 2001.

[8] Iai S, Ichii K. Performance based design for port structures. In: Proceedings of UJNR 30th joint meeting of United States-Japan panel on wind and seismic effects, Gaithersburg; 1998. p. 84-96.

[9] Itasca. FLAC Fast Lagrangian analysis of Continua v. 5.0. User's Manual; 2005.

[10] Lancellotta R. Analytical solution of passive earth pressure. Géotechnique 2002;52(8):617-9. 
[11] Lancellotta R. Lower-bound approach for seismic passive earth resistance. Géotechnique 2007;57(3):319-21.

[12] Madabhushi SPG, Zeng X. Seismic response of flexible cantilever retaining walls with dry backfill. Geomechanics and Geoengineering 2006;1(4):275-89.

[13] Madabhushi SPG, Zeng X. Simulating seismic response of cantilever retaining walls. Journal of Geotechnical and Geoenvironmental Engineering 2007: 539-49.

[14] Masing G. Eigenspannungen und Verfertigung bim Messing. In: Proceedings of the 2nd international congress on applied mechanics, Zurich; 1926.

[15] Mononobe N, Matsuo H. On the determination of earth pressure during earthquake. Proceedings of the 2nd World Engineering Conference 1929;9: 177-85.

[16] Neelakantan G, Budhu M, Richards R. Balanced seismic design of anchored retaining walls. Journal of Geotechnical Engineering 1992;118(6):873-88.

[17] Newmark NM. Effects of earthquakes on dams and embankments. Géotechnique 1965:15(2):139-60.

[18] Okabe S. General theory of earth pressure and seismic stability of retaining wall and dam. Journal of Japanese Society of Civil Engineering 1924;12:1.
[19] Richards R, Elms DG. Seismic behavior of gravity retaining walls. Journal of Geotechnical Engineering Division, 105; 449-64 ASCE.

[20] Richards R, Elms DG. Seismic passive resistance of tied-back walls. Journal of Geotechnical Engineering 1992;118(7):996-1011.

[21] Steedman RS. Seismic design of retaining walls. Proceedings of the Institution of Civil Engineers. Geotechnical Engineering 1998;131:12-22.

[22] Towhata I, Islam MS. Prediction of lateral displacement of anchored bulkheads induced by seismic liquefaction. Soils and Foundations 1987;27(4):137-47.

[23] Vucetic M, Dobry R. Effect of soil plasticity on cyclic response. Journal of Geotechnical and Geoenvironmental Engineering 1991;117(1):89-107.

[24] Whitman RV. Seismic design and behaviour of gravity retaining walls. Design and Performance of Earth Retaining Structures, 25. ASCE, Geotech. Special Publication; 817-42.

[25] Zeng X.. Modelling the behaviour of quay walls in earthquakes. PhD thesis. Cambridge, UK: University of Cambridge; 1990.

[26] Zienkiewicz OC, Bianic N, Shen FO. Earthquake input definition and the transmitting boundary condition. In: St. Doltnis I, editor. Conf: Advances in computational non-linear mechanics; 1988. p. 109-38. 